\title{
Hydraulics of smoothly streamlined Venturi channels of critical depth
}

\author{
Andrey Zuikov ${ }^{1,2, *}$ and Tatiana Suehtina ${ }^{2}$ \\ ${ }^{1}$ Moscow State University of Civil Engineering, 26 Jaroslavskoe Shosse, Moscow, 129337, Russia \\ ${ }^{2}$ Moscow Automobile and Road Construction State Technical University, 64 Leningradsky prospect, \\ Moscow, 125319, Russia
}

\begin{abstract}
The article relates to the field of hydraulics and engineering hydrology and is devoted to a study of fluid flow in a non-flooded Venturi channel. The purpose of the work is improvement of methods for calculating profiles and hydraulic characteristics of a Venturi flumes used for measurement of water flow rates in open channels and rivers. Research methods are analytical with experimental verification. A functional relationship is obtained between the Froude number in an arbitrary section of the Venturi channel and its width normalized by the width of the critical section. It is established that within a rectilinear gorge portion of a Venturi channel, the flow is unstable, which is related to the proximity of its parameters to the critical ones. The method of optimization of a profile of a Venturi channel with a dividing cross-section in a gorge that does not contain empirical coefficients is considered. It is shown that the proposed method allows determining all main geometric parameters and hydraulic characteristics of the Venturi flume, including its flow rate coefficient, distribution of depths and flow velocities along the length of a flume with a relative error of $\pm 1 \%$.
\end{abstract}

\section{Introduction}

The priority of using water resources for domestic water supply declared in the Water Code of the Russian Federation and the attitude to water as a strategic resource of the country allow us to claim that control to the water consumption from natural sources and sewage return to the environment is the most important State task. Requirements for accounting for water use are approved by Decrees of the Government of the Russian Federation of 12.02.1999 No. 167, of 10.04.2007 No. 219 and of 29.07.2013 No. 644 and also by Order of the Ministry of Natural Resources of the Russian Federation of 08.07.2009 No. 205. These documents determine that all enterprises in Russia, without exception, must be equipped with measuring stations to determine the volume of clean water consumption and wastewater discharge. According to [1-3], the main current State Standards of Russian Federation that regulate the measurement of open flow rate are two documents: MI 2220-13 "The Flow Rate and Volume of the Waste Fluid. Gauging Procedure in Non-pressure Water Conduits by the Level of Filling with Preliminary Calibration of the Measuring Section"

\footnotetext{
*Corresponding author: zuykov54@mail.ru
} 
and MI 2406-97 "Liquid Flow in Open Channels of Water Supply and Sewerage Systems. Gauging Procedure with the Use of Standard Weirs and Flumes". These documents recognize the Venturi channels [1-3] as effective means of determining of open flow rates in natural watercourses, channels for land reclamation, household water supply and sewerage. A useful quality of the Venturi channels is the ability of passing significantly polluted flows containing suspended solids, sand, branches, logs and other garbage.

Venturi flumes are non-prismatic channels with vertical walls, at the entrance section is narrowed downstream, followed by a straightforward gorge and gradually expanding downstream portion. Venturi channels with a horizontal bottom, with critical depth in the gorge and with free (not flooded) fluid outflow [4-15], where downstream water level $\left(h_{0}\right)$ variation has no effect on the flow rate $(Q)$, are considered classic. Classical Venturi channels, whose smooth longitudinal profile and calculation methods are regulated by the Russian standard MI 2406-97, are applied where it is necessary to provide the measurement of flow rates with an error not exceeding $\pm 5 \%$, for example, in water supply and sewerage systems. On large channels and natural watercourses it is often necessary to simplify the Venturi channels profile, making the mating of adjacent areas flat vertical walls at an angl. This leads to significant hydraulic losses in the measuring area from upstream pool to the gorge of Venturi cannel, and, consequently, this leads to the incorrect application of the regulations of the standard MI 2406-97. Such channels need additional calibration of the measuring sections in place. But the hydraulics of the classical Venturi channels of critical depth according to the regulations of the standard MI 2406-97, as our studies show [16-18], can be improved by practically eliminating hydraulic losses if the channel is made extremely streamlined. In papers [16-18], an analytic formula, free of empirical coefficients, is derived to compute the full-capacity discharge of the Venturi channels (flumes), both in accordance with the MI 2406-97 standard and the streamlined ones, which makes it possible to increase the accuracy of calculating to the errors $\pm 1 \%$. In general, these studies suggest that the open Venturi channel is advisable to perform as a pressure Venturi nozzle, where a smooth confuser ends at a separate cross-section in the gorge, after which followed a diffuser. This work is devoted to verification of this provision. The purpose of the work is improvement of methods for calculating profiles and hydraulic characteristics.

\section{Methods}

Research methods are analytical with experimental verification by data of papers [16-18].

\section{Results and Discussion}

\subsection{Fluid motion in a non-prismatic smoothly changing channel}

Let us consider a smoothly changing fluid flow in a non-prismatic channel with a variable smoothly changing width $b$, vertical walls and a bottom slope $i$. The equation of changing in the amount of fluid motion over an infinitely small channel length $d x$ (Fig. 1) under the action of external forces per unit of time has the form [19]

$$
\rho Q \alpha_{0}(V+d V)-\rho Q \alpha_{0} V=\Sigma F,
$$

where $\rho$ is density of the liquid; $Q$ is flow rate; $\alpha_{0}$ is the Boussinesq coefficient; $V$ is the average flow velocity at the inlet of the selected section of the channel; $V+d V$ is the average flow velocity at the exit of the selected channel section; $\Sigma F$ is the sum of external forces acting on the selected mass of fluid in the $d x$ segment in projection on the direction of mo- 
tion

$$
\Sigma F=P \omega-(P+d P)(\omega+d \omega)+R_{x}+G \sin \beta-\tau_{0} \chi d x
$$

$P$ is pressure at the center of gravity of the cross-section with area $\omega=b h$ at the inlet of the selected channel segment; $h$ is depth of the stream at the inlet of the selected segment; $P+$ $d P$ is pressure at the center of gravity of the section with area $\omega+d \omega$ at the output of the selected segment; $R_{x}$ is the reaction force of the two side walls of the non-prismatic channel in the projection on the direction of flow motion (this force is the inverse of the fluid pressure force on the walls in the same projection); $G$ is weight of the liquid on the segment $d x ; \beta$ is the angle of inclination of the bottom of the channel to the horizon; $\tau_{0}$ is stresses of hydraulic friction on the walls and bottom of the channel; $\chi$ is wetted perimeter.
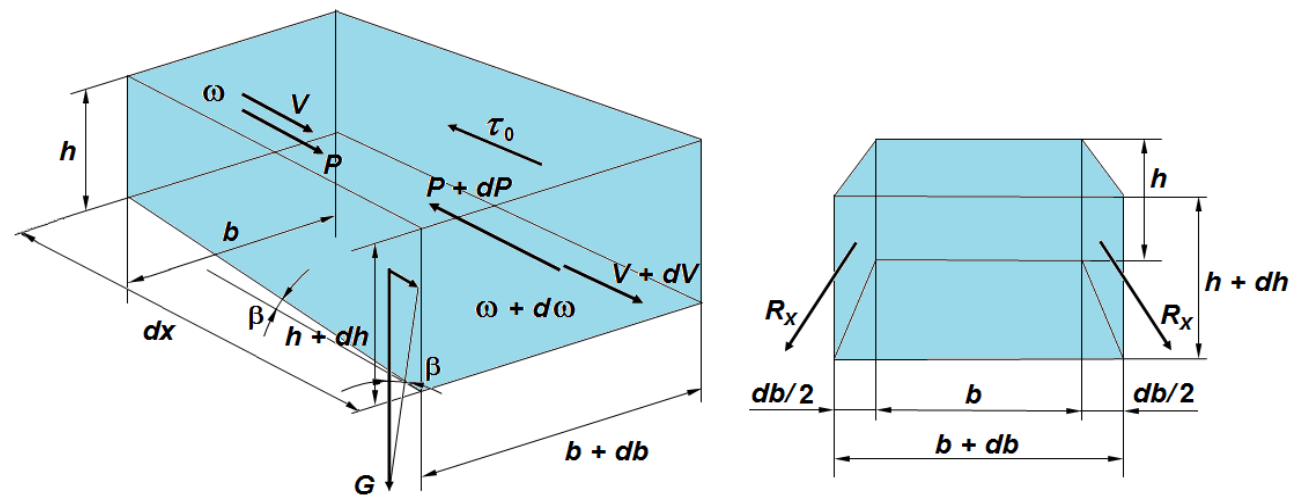

Fig. 1. The computed scheme of the smoothly changing fluid motion in a non-prismatic channel with vertical walls.

Such kind of an approach to the study of an uneven open flow is called "dynamic". It was previously used to analyze the movement of fluid in wide prismatic cannels and weirs with a wide threshold [19-21]. The "dynamic" approach based on the equation of momentum, in our opinion, should have an advantage in comparison with the traditional "energy" approach, based on the equations of Bernoulli and Charnomsky. The equation of momentum of forces (1) explicitly takes into account the mass of a moving fluid, that is, the inertia of motion, while there is no mass in the Bernoulli and Charnomsky equations. Thus, it is impossible to conclude from the equations of Bernoulli and Charnomsky that the flow has time to change between the control cross-sections.

According to the computed scheme in Fig. 1 the reaction of two side walls of the channel in the projection on the flow direction can be defined as

$$
R_{x}=2 P_{x} \omega_{x}
$$

where $P_{x}$ is the pressure at the center of gravity of the side lateral surface

$$
P_{x}=\frac{1}{2} \rho g \frac{2 h+d h}{2} ;
$$

$\omega_{x}$ is the area of the projection of the surface of the side wall on the cross-section of the flow

$$
\omega_{x}=\frac{1}{2} h \frac{d b}{2}+\frac{1}{2}(h+d h) \frac{d b}{2} .
$$

Then, neglecting small of the second and third orders, we write 


$$
R_{x}=\rho g \frac{h^{2}}{2} d b
$$

The weight of a liquid on a selected segment, equal to the product of the volume of the prism it occupies

$$
W=\frac{1}{3}[\omega+\sqrt{\omega(\omega+d \omega)}+\omega+d \omega] d x \cos \beta,
$$

density $\rho$ and gravity acceleration $g=9.81 \mathrm{~m} / \mathrm{sec}^{2}$, multiplied by the sine of the inclination angle of the channel bottom to the horizon, is equal to the projection of the force from the weight of the liquid on the flow direction

$$
G \sin \beta=\rho g \omega d x \cos \beta \sin \beta \text {. }
$$

Since the sine of the inclination angle of the channel bottom to the horizon at $\beta \rightarrow 0$ is equal to the slope of the channel bottom $\beta=\sin \beta=i$ and

$$
\cos \beta \sin \beta=\frac{1}{2} \sin 2 \beta=\beta=i,
$$

then

$$
G \sin \beta=\rho g \omega i d x .
$$

Substituting now the values of the acting forces into equation (2) and then to the equation of momentum (1), and also noting that $\omega=b h$ and $P=\rho g h / 2$, we get

$$
\rho Q \alpha_{0} d V=-\rho g \omega d h+\rho g \omega i d x-\tau_{0} \chi d x .
$$

But, the average flow velocity at the inlet of the selected channel segment is equal to

$$
V=\frac{Q}{\omega}
$$

then, dividing expression (3) by the product $\rho g \omega d x$, we can write

$$
-\frac{\alpha_{0} Q^{2}}{g \omega^{3}} \cdot \frac{d \omega}{d x}=-\frac{d h}{d x}+i-\frac{\tau_{0}}{\rho g R},
$$

here $R$ is the hydraulic radius, $R=\omega / \chi$.

If we consider that for a non-prismatic canal with a width " $b$ " by water level, we have

$$
\frac{d \omega}{d x}=b \frac{d h}{d x}+\frac{\partial \omega}{\partial x}
$$

where $\partial \omega / \partial x$ is the partial derivative of the change in the area of the flow live cross-section, which does not depend on the change in its depth, we finally find

$$
\frac{d h}{d x}=\frac{i+\frac{\alpha_{0} Q^{2}}{g \omega^{3}} \cdot \frac{\partial \omega}{\partial x}-\frac{\tau_{0}}{\rho g R}}{1-\frac{\alpha_{0} Q^{2} b}{g \omega^{3}}} .
$$


It can be seen that Eq. (5) is a well-known differential equation of the smoothly changing fluid motion in an open channel that is obtained within the framework of the classical "energetic" approach from the Bernoulli and Charnomsky equations. However, in the classical equation, the Coriolis kinetic energy coefficient $\alpha$ applies, while in Eq. (5) the Boussinesq momentum coefficient $\alpha_{0}$ is used. The coefficients are close in magnitude, for turbulent open flows, they are usually taken equal to: $\alpha_{0}=1.02 \div 1.03$ and $\alpha=1.06 \div 1.09$. In engineering calculations, where the allowable error is $\pm 5 \%$, the Boussinesq correction can be neglected, assuming it to be equal to $\alpha_{0}=1$. Thus, with the "dynamic" and "energetic" approaches, the differential equation of the smoothly changing fluid movement in an open channel remains the same. This is determined by the model of a smoothly changing movement, in which the inertia factor of the flow is lost. When this factor is lost, the flow always has time to rearrange itself between the cross-sections in accordance with the change in the non-prismatic channel.

Since the depth-independent partial derivative of the function of changing the crosssectional area of the flow for a non-prismatic channel of variable width $b$ and with vertical walls is equal to

$$
\frac{\partial \omega}{\partial x}=h \frac{d b}{d x},
$$

then equation (5) can be rewritten in the form

$$
\frac{d h}{d x}=\frac{i+\frac{\alpha_{0} Q^{2} h}{g \omega^{3}} \cdot \frac{d b}{d x}-\frac{\tau_{0}}{\rho g R}}{1-\frac{\alpha_{0} Q^{2} b}{g \omega^{3}}},
$$

and for a channel with a horizontal bottom $(i=0)$ it reduces to equality

$$
\frac{d h}{d x}=\frac{\frac{\alpha_{0} Q^{2} h}{g \omega^{3}} \cdot \frac{d b}{d x}-\frac{\tau_{0}}{\rho g R}}{1-\frac{\alpha_{0} Q^{2} b}{g \omega^{3}}} .
$$

\subsection{Fluid motion in Venturi channels of critical depth}

Let us now turn to an analysis of formula (6), which reflects the hydraulics of flows in nonprismatic channels with vertical walls and a horizontal bottom, to which the Venturi channels belong.

If the Froude number is written as [16-18]

$$
\mathrm{Fr}=\frac{V}{\sqrt{g h}},
$$

then for $\omega=b h$, according to equality (4) 


$$
\frac{\alpha_{0} Q^{2} b}{g \omega^{3}}=\frac{\alpha_{0} V^{2}}{g h}=\alpha_{0} \mathrm{Fr}^{2}
$$

Putting the hydraulic losses associated with the roughness of the channel walls negligibly small, we bring equation (6) to the form

$$
\frac{d h}{d x}=\frac{\alpha_{0} \mathrm{Fr}^{2}}{1-\alpha_{0} \mathrm{Fr}^{2}} \cdot \frac{h}{b} \cdot \frac{d b}{d x}
$$

The analysis shows: at the inlet portion of the Venturi flume, where there is a calm subcritical flow with Froude numbers less than unity $(\mathrm{Fr}<1)[18]$ the denominator of Eq. (9) is greater than zero, therefore, in this area the narrowing of the channel when $d b / d x<0$, causes a decrease in the depth of the stream $(d h / d x<0)$, a the expansion of the channel $(d b / d x>0)$ causes an increase in the depth $(d h / d x>0)$; in the bottom diffuser of the flume, where a stormy supercritical flow with Froude numbers greater than unity $(\mathrm{Fr}>1)$ [18] is observed, the denominator of Eq. (9) is less than zero, the depth of such flow decreases $(d h / d x<0)$ when the channel is expanded $(d b / d x>0)$, and the increase in depth $(d h / d x>0)$ occurs at its narrowing $(d b / d x<0)$. The flow transition from calm to stormy takes place in the cross-section with a critical depth [22]

$$
h_{c}=\sqrt[3]{\frac{\alpha_{0} Q^{2}}{g b_{c}^{2}}},
$$

where $b_{c}$ is the Venturi channel width in the critical cross-section (Fig. 2). In a not flooded Venturi channels, the transition from calm to stormy flow is irreversible, and the flow depth from the upper to lower pools, according to the data of [18], is constantly decreasing, converting to kinetic energy.

Let's express the derivative $d h / d x$ in terms of the Froude number. According to equalities (7), (4) and $\omega=b h$ we find

$$
\frac{d \mathrm{Fr}}{d x}=\frac{Q}{\sqrt{g}} \frac{d}{d x}\left(\frac{1}{b h^{1,5}}\right)=-\frac{Q}{b^{2} h^{1,5} \sqrt{g}} \cdot \frac{d b}{d x}-\frac{3 Q}{2 b h^{2,5} \sqrt{g}} \cdot \frac{d h}{d x}=-\frac{\mathrm{Fr}}{b} \cdot \frac{d b}{d x}-\frac{3 \mathrm{Fr}}{2 h} \cdot \frac{d h}{d x},
$$

whence

$$
\frac{d h}{d x}=-\frac{2 h}{3 \mathrm{Fr}} \cdot \frac{d \mathrm{Fr}}{d x}-\frac{2 h}{3 b} \cdot \frac{d b}{d x} .
$$

Substituting the obtained value of the $d h / d x$ derivative into expression (9), we come to equation

$$
\frac{\alpha_{0} \operatorname{Fr}^{2}-1}{\alpha_{0} \operatorname{Fr}^{2}\left(\alpha_{0} \operatorname{Fr}^{2}+2\right)} \cdot d\left(\alpha_{0} \operatorname{Fr}^{2}\right)=\frac{d b}{b}
$$



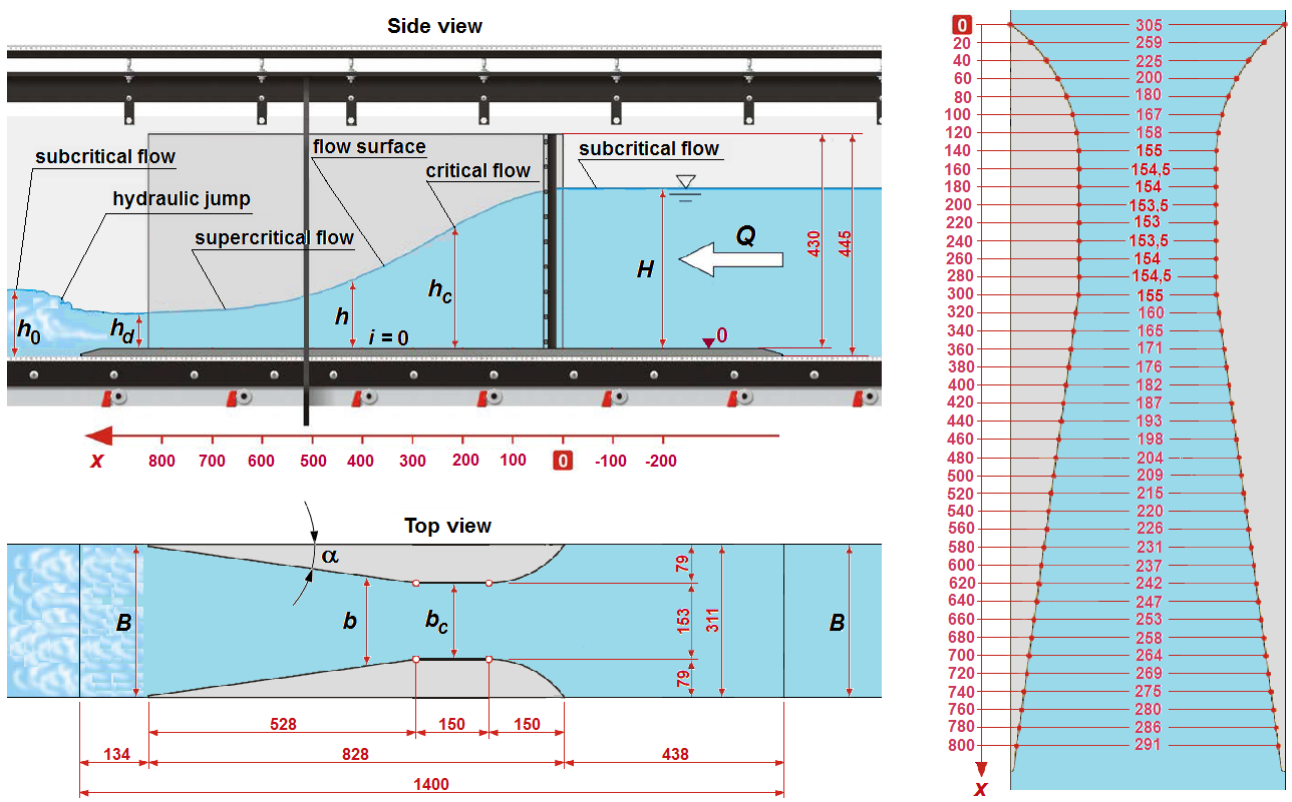

Fig. 2. Laboratory model of Venturi flume (measurements in millimeters).

Since within the gorge the flow passes through a critical cross-section with width $b=$ $b_{c}$, depth $h=h_{c}$ and area $\omega=b_{c} h_{c}$ (cf. Fig. 2), where, following (5), (6) and (9), it's possible write

$$
1-\frac{\alpha_{0} Q^{2} b}{g \omega^{3}}=1-\alpha_{0} \mathrm{Fr}^{2}=0,
$$

then taking these conditions as the boundary ones, as a result of integration (10) we find

$$
\frac{b}{b_{c}}=\sqrt{\frac{\left(\alpha_{0} \mathrm{Fr}^{2}+2\right)^{3}}{27 \alpha_{0} \mathrm{Fr}^{2}}} \text {. }
$$

Formula (11) is convenient for solving the direct problem of determining the longitudinal variation of the width of the Venturi channel $b(x)$ if the width of the gorge $b_{c}$ is known and the function $\operatorname{Fr}(x)$ is given. For the inverse problem of determining the change in flow characteristics along the channel length, we will bring formula (11) to the form

$$
27\left(\frac{b}{b_{c}}\right)^{2} \alpha_{0} \mathrm{Fr}^{2}=\left(\alpha_{0} \mathrm{Fr}^{2}+2\right)^{3} .
$$

Putting $\alpha_{0} \mathrm{Fr}^{2}+2=y$, we reduce Eq. (12) to cubic Cardano equation $y^{3}+p y+q=0$ [23]

$$
y^{3}-27\left(\frac{b}{b_{c}}\right)^{2} y+54\left(\frac{b}{b_{c}}\right)^{2}=0,
$$

the coefficients of which equal 


$$
p=-27\left(\frac{b}{b_{c}}\right)^{2}<0 \quad \text { and } \quad q=54\left(\frac{b}{b_{c}}\right)^{2}
$$

But if the coefficient $p<0$ and the sum

$$
\left(\frac{p}{3}\right)^{3}+\left(\frac{q}{2}\right)^{2}=\left[-\frac{27}{3}\left(\frac{b}{b_{c}}\right)^{2}\right]^{3}+\left[\frac{54}{2}\left(\frac{b}{b_{c}}\right)^{2}\right]^{2}=27^{2}\left(\frac{b}{b_{c}}\right)^{4}\left[1-\left(\frac{b}{b_{c}}\right)^{2}\right]
$$

is also less than zero, as $b>b_{c}$, then the cubic Eq. (13) has a trigonometric solution with three real roots

$$
y_{1,2,3}=2 \sqrt{-\frac{p}{3}} \cos \left(\frac{\theta+2 n \pi}{3}\right)=6 \frac{b}{b_{c}} \cos \left(\frac{\theta+2 n \pi}{3}\right), \quad(n=0,1,2),
$$

where

$$
\cos \theta=-\frac{q}{2} \sqrt{-\left(\frac{3}{p}\right)^{3}}=-\frac{b_{c}}{b}
$$

The analysis showed that the solutions of the cubic Eq. (13) are:

- for the upper inlet section of the Venturi flume with $0<\mathrm{Fr}<1$ - the third root for $n=2$

$$
y=\alpha_{0} \operatorname{Fr}^{2}+2=6 \frac{b}{b_{c}} \cos \left\{\frac{1}{3}\left[\arccos \left\{-\frac{b_{c}}{b}\right)+4 \pi\right]\right\}=6 \frac{b}{b_{c}} \sin \left[\frac{1}{3} \arcsin \left(\frac{b_{c}}{b}\right)\right]
$$

or

$$
\alpha_{0} \operatorname{Fr}^{2}=\frac{\alpha_{0} Q^{2}}{g b^{2} h^{3}}=6 \frac{b}{b_{c}} \sin \left[\frac{1}{3} \arcsin \left(\frac{b_{c}}{b}\right)\right]-2=8 \frac{b}{b_{c}} \sin ^{3}\left[\frac{1}{3} \arcsin \left(\frac{b_{c}}{b}\right)\right]
$$

- for the down outlet section with Fr $>1$ - the first root for $n=0$

$$
y=\alpha_{0} \mathrm{Fr}^{2}+2=6 \frac{b}{b_{c}} \cos \left[\frac{1}{3} \arccos \left(-\frac{b_{c}}{b}\right)\right]
$$

or

$$
\alpha_{0} \mathrm{Fr}^{2}=\frac{\alpha_{0} Q^{2}}{g b^{2} h^{3}}=6 \frac{b}{b_{c}} \cos \left[\frac{1}{3} \arccos \left(-\frac{b_{c}}{b}\right)\right]-2=8 \frac{b}{b_{c}} \cos ^{3}\left[\frac{1}{3} \arccos \left(-\frac{b_{c}}{b}\right)\right] .
$$

In this case, in upstream and downstream pools of the Venturi flume with channel width B (cf. Fig. 2), we obtain

$$
\alpha_{0} \mathrm{Fr}_{u}^{2}=\frac{\alpha_{0} Q^{2}}{g B^{2} H^{3}}=8 \frac{B}{b_{c}} \sin ^{3}\left[\frac{1}{3} \arcsin \left(\frac{b_{c}}{B}\right)\right]
$$




$$
\alpha_{0} \mathrm{Fr}_{d}^{2}=\frac{\alpha_{0} Q^{2}}{g B^{2} h_{d}^{3}}=8 \frac{B}{b_{c}} \cos ^{3}\left[\frac{1}{3} \arccos \left(-\frac{b_{c}}{B}\right)\right],
$$

here $\mathrm{Fr}_{u}$ and $\mathrm{Fr}_{d}$ are the Froude numbers in the upper and lower pools of the Venturi flume; $H$ is the flow depth in the upstream pool (cf. Fig. 2); $h_{d}$ is the depth of the flow at the outlet from the Venturi flume into the downstream channel.

Let's express the capacity discharge through the flow coefficient $m$

$$
Q=m B \sqrt{2 g} H^{1.5}
$$

whence, according to (16), we find

$$
m=\sqrt{0.5} \operatorname{Fr}_{u}=2 \sqrt{\frac{B}{b_{c}}} \sin ^{1.5}\left[\frac{1}{3} \arcsin \left(\frac{b_{c}}{B}\right)\right] .
$$

Formula (19) was obtained by us earlier in papers [16-18] and underwent experimental verification, which showed that the error in calculations by it is $\pm 1 \%$.

Using formulas (8), (14) - (17) it is possible to determine also all the main hydraulic characteristics of the flow in the Venturi channels:

- change of flow depth along the flume

for the upper inlet section with $\mathrm{Fr}<1$

$$
\frac{h}{H}=\frac{B}{b} \cdot \frac{\sin \left[\frac{1}{3} \arcsin \left(\frac{b_{c}}{B}\right)\right]}{\sin \left[\frac{1}{3} \arcsin \left(\frac{b_{c}}{b}\right)\right]}
$$

for the down outlet section with $\mathrm{Fr}>1 \frac{h}{H}=\frac{B}{b} \cdot \frac{\sin \left[\frac{1}{3} \arcsin \left(\frac{b_{c}}{B}\right)\right]}{\cos \left[\frac{1}{3} \arccos \left(-\frac{b_{c}}{b}\right)\right]}$,

whence it follows that from the entrance of Venturi channel to the critical cross-section the depth of the flow falls arom $H$ to the value

$$
\frac{h_{c}}{H}=2 \frac{B}{b_{c}} \sin \left[\frac{1}{3} \arcsin \left(\frac{b_{c}}{B}\right)\right],
$$

after the critical cross-section, the decrease the flow depth continues and at the exit from the Venturi flume to the downstream channel the flow depth is

$$
\frac{h_{d}}{H}=\frac{\sin \left[\frac{1}{3} \arcsin \left(\frac{b_{c}}{B}\right)\right]}{\cos \left[\frac{1}{3} \arccos \left(-\frac{b_{c}}{B}\right)\right]} ;
$$

- change of flow velocities along the flume 
for the upper inlet section with Fr $<1 \frac{V}{\sqrt{2 g H}}=\sqrt{2 \frac{h_{c}}{H}} \sin \left[\frac{1}{3} \arcsin \left(\frac{b_{c}}{b}\right)\right]$;

for the down outlet section with Fr $>1 \frac{V}{\sqrt{2 g H}}=\sqrt{2 \frac{h_{c}}{H}} \cos \left[\frac{1}{3} \arccos \left(-\frac{b_{c}}{b}\right)\right]$.

In the derivation of formulas (23) and (24), $\alpha_{0}=1$ was taken. Raising equalities (23) and (24) in the square, one can obtain the velocity head of the flow, which in the critical cross-section of the Venturi flume with width $b_{c}$ is $0.5 h_{c}$.

The considered flow is observed in the Venturi channel with free (not flooded) fluid outflow. However, at a normal downstream depth $h_{0}$ (cf. Fig. 2) is greater than the conjugate depth

$$
h_{0}>\frac{h_{d}}{2}\left[\sqrt{1+8 \frac{h_{\kappa}^{3}}{h_{d}^{3}}}-1\right]
$$

there will be a flooded mode of expiration, which according to the State Standard of Russia MI 2406-97 is considered inadmissible. Since the critical depth in a channel of rectangular cross-section with width $B$ is

$$
h_{\kappa}=\sqrt[3]{\frac{\alpha_{0} Q^{2}}{g B^{2}}}
$$

whence

$$
\frac{h_{\kappa}^{3}}{h_{d}^{3}}=\frac{\alpha_{0} Q^{2}}{g B^{2} h_{d}^{3}}=\alpha_{0} \mathrm{Fr}_{d}^{2},
$$

then, following (17) and (22), in order to ensure unflooded outflow, the condition should be observed

$$
\frac{h_{0}}{H} \leq \frac{\sin \left[\frac{1}{3} \arcsin \left(\frac{b_{c}}{B}\right)\right]}{2 \cos \left[\frac{1}{3} \arccos \left(-\frac{b_{c}}{B}\right)\right]}\left\{\sqrt{1+64 \frac{B}{b_{c}} \cos ^{3}\left[\frac{1}{3} \arccos \left(-\frac{b_{c}}{B}\right)\right]}-1\right\}
$$

\subsection{Verification of the method for calculating flow characteristics in Venturi channel}

Let us check how adequately the computed formulas obtained correspond to actually observed flows. To do this, let's compare the computed values with the analogous measured characteristics of the model of Venturi channel shown in Fig. 2. The description of the used experimental equipment, devices, measuring means and instruments, as well as a description of the methodology of the experimental investigations are given in detail in our previously published papers [16-18]. In these papers, the empirical material is shown in a wide range of changes in the flow rates the Venturi channel (from $Q=5$ to $116 \mathrm{~m} 3 / \mathrm{h}$ ). We do not find it advisable to cover the whole studied range, we confine ourselves to one characteris- 
tic mode, for example, with the flow rate $Q=90.14 \mathrm{~m}^{3} / \mathrm{h}$ and with head in the upstream pool $H=0.20012 \mathrm{~m}$.

For the investigated Venturi flume (cf. Fig. 2) with width of the upstream channel $B=$ $311 \mathrm{~mm}$ and a gorge width $b_{c}=153 \mathrm{~mm}$, we obtain the following calculated value of the flow coefficient

$$
m=2 \sqrt{\frac{B}{b_{c}}} \sin ^{1.5}\left[\frac{1}{3} \arcsin \left(\frac{b_{c}}{B}\right)\right]=2 \sqrt{\frac{311}{153}} \sin ^{1.5}\left[\frac{1}{3} \arcsin \left(\frac{153}{311}\right)\right]=0.20094 .
$$

Substituting the value of $m$, and also the head $H=0.20012 \mathrm{~m}$ in (18), as a result we find $Q=m B \sqrt{2 g} H^{1.5}=0.20094 \cdot 0.311 \sqrt{2 \cdot 9.81} \cdot 0.20012^{1.5}=0.02478 \mathrm{~m}^{3} / \mathrm{sec}=89.21 \mathrm{~m}^{3} / \mathrm{h}$.

It can be seen that the computed value of the flow rate is only $1 \%$ different from the actual value, determined as a result of direct measurements.

Let us now compare the measured values of the relative depths $h / H$, flow velocities along the length of the Venturi channel

and Froude numbers

$$
\frac{V}{\sqrt{2 g H}}=\frac{Q}{b h \sqrt{2 g H}}
$$

$$
\mathrm{Fr}=\frac{V}{\sqrt{g h}}=\frac{Q}{\sqrt{g} b h^{1.5}}
$$

with their computed values by the formulas (20), (21), (23), (24), and also (14) and (15), reduced to the form

$$
\begin{aligned}
& \text { for the upper inlet section with } \mathrm{Fr}<1 \quad \mathrm{Fr}=2 \sqrt{2 \frac{b}{b_{c}}} \sin 1.5\left[\frac{1}{3} \arcsin \left(\frac{b_{c}}{b}\right)\right] \\
& \text { for the down outlet section with } \mathrm{Fr}>1 \quad \mathrm{Fr}=2 \sqrt{2 \frac{b}{b_{c}}} \cos ^{1.5}\left[\frac{1}{3} \arccos \left(-\frac{b_{c}}{b}\right)\right] .
\end{aligned}
$$

The results of experiments and calculations are summarized in Table 1 and shown in Fig. 3, in which the longitudinal and transverse coordinates in the plane of the bottom of the flume are labeled as $x$ and $y$.

Table 1. Experimental and calculated flow characteristics in the model of Venturi channel.

\begin{tabular}{|c|c|c|c|c|c|c|c|c|c|}
\hline \multicolumn{3}{|c|}{ Experimental data } & \multicolumn{3}{c|}{ Experimental data processing } & \multicolumn{3}{c|}{ Calculated values } \\
\hline$x, \mathrm{~mm}$ & $b, \mathrm{~mm}$ & $h, \mathrm{~mm}$ & $V, \mathrm{~m} / \mathrm{sec}$ & $h / H$ & $V /(2 g H)^{0,5}$ & Fr & $h / H$ & $V /(2 g H)^{0,5}$ & Fr \\
\hline-200 & 311 & 200.12 & 0.4023 & 1.0000 & 0.20303 & 0.2871 & 1.0000 & 0.20094 & 0.2842 \\
\hline-100 & 311 & 199.43 & 0.4037 & 0.9966 & 0.20374 & 0.2886 & 1.0000 & 0.20094 & 0.2842 \\
\hline 0 & 305 & 196.97 & 0.4168 & 0.9843 & 0.21034 & 0.2998 & 0.9982 & 0.20525 & 0.2905 \\
\hline 100 & 167 & 184.63 & 0.8121 & 0.9226 & 0.40983 & 0.6034 & 0.8436 & 0.44357 & 0.683 \\
\hline 200 & 153.5 & 154.47 & 1.0560 & 0.7719 & 0.53293 & 0.8578 & 0.7254 & 0.56123 & 0.9319 \\
\hline 300 & 155 & 119.74 & 1.3491 & 0.5983 & 0.68085 & 1.2447 & 0.6273 & 0.64270 & 1.1476 \\
\hline 400 & 182 & 94.38 & 1.4577 & 0.4716 & 0.73565 & 1.5149 & 0.4450 & 0.77161 & 1.6358 \\
\hline 500 & 209 & 77.66 & 1.5427 & 0.3881 & 0.77853 & 1.7674 & 0.3634 & 0.82278 & 1.9302 \\
\hline 600 & 237 & 65.07 & 1.6236 & 0.3252 & 0.81939 & 2.0322 & 0.3081 & 0.85571 & 2.1800 \\
\hline 700 & 264 & 55.13 & 1.7204 & 0.2755 & 0.86822 & 2.3393 & 0.2696 & 0.87792 & 2.3911 \\
\hline 800 & 291 & 47.87 & 1.7975 & 0.2392 & 0.90712 & 2.6230 & 0.2400 & 0.89461 & 2.5823 \\
\hline 900 & 311 & 44.45 & 1.8111 & 0.2221 & 0.91400 & 2.7425 & 0.2221 & 0.90456 & 2.7142 \\
\hline
\end{tabular}




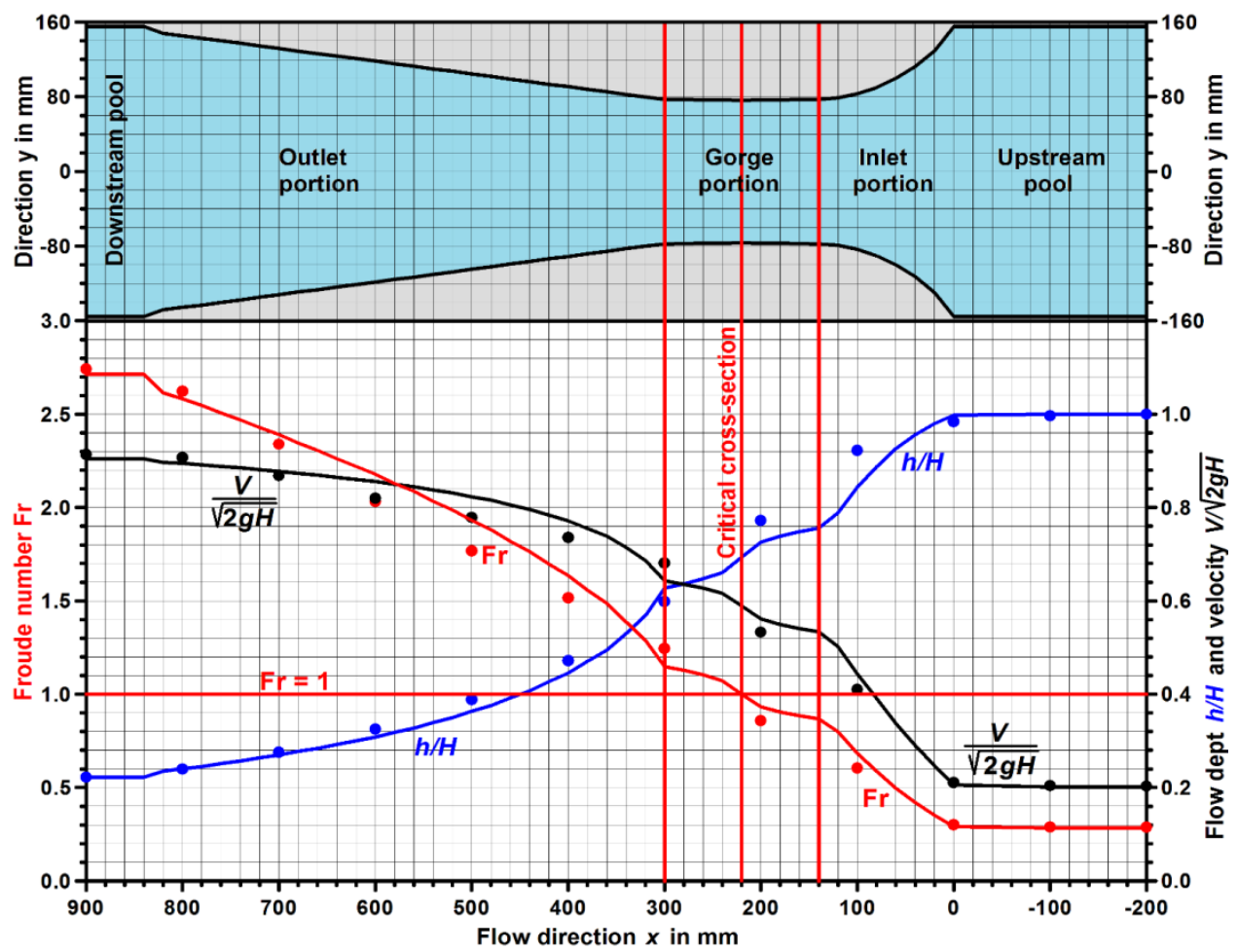

Fig. 3. Change in depth of flow, velocities and Froude numbers along the length of the investigated Venturi channel: experimental values are shown by points, computed - solid lines.

For the investigated Venturi flume, the unflooded flow condition (25) is determined by the inequality

$$
\frac{h_{0}}{H} \leq \frac{\sin \left[\frac{1}{3} \arcsin \left(\frac{153}{311}\right)\right]}{2 \cos \left[\frac{1}{3} \arccos \left(-\frac{153}{311}\right)\right]}\left\{\sqrt{1+64 \frac{311}{153} \cos ^{3}\left[\frac{1}{3} \arccos \left(-\frac{153}{311}\right)\right]}-1\right\}=0.75 .
$$

The obtained calculated value of $h_{0} / H$ exactly corresponds to the recommendations of the Russian State Standard MI 2406-97.

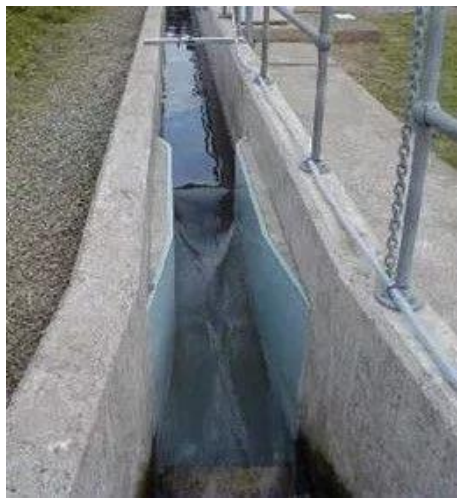

Fig. 4. Venturi flume on irrigation cannel (view from downstream pool). 
It can be seen that the computed values of the flow characteristics in the investigated Venturi channel are in good agreement with the experimental data in order to assume the considered method of hydraulic computation to be completely satisfying the problem of analyzing flows in the Venturi channels and to use it in subsequent investigations. We consider it necessary to pay attention to the fact that if all the flow characteristics at the inlet and outlet sections Venturi channel, made according by State Standard MI 2406-97, change smoothly, then at the gorge this occurs in an wave-shaped. This was noted in [18], where it is said about unstable fluid motion at the gorge portion of substantial length, which is accompanied by unjustified hydraulic losses. An illustration of this fact is also the photograph in Fig. 4, where it is possible to observe waves on the flow surface in the gorge of the Venturi channel. The phenomenon is related to the proximity of the flow parameters to the critical ones with Froude numbers from $\mathrm{Fr}=0.85$ to $\mathrm{Fr}=1.15$. In this regard, we find it impractical to perform the gorge in a form of a straight channel. Moreover, then longer the gorge, the more unstable the nature of the flow observed in it will affect the hydraulics of the water meter channel as a whole, and the less its characteristics will be amenable to theoretical calculation, necessitating its additional experimental calibration. By analogy with pressurized water-measuring nozzles of the Venturi, we find it expedient to perform water meter Venturi channels with critical cross-section at a gorge as a dividing cut. In the gorge, when the flow passes through $\mathrm{Fr}=1$, the increase of Froude numbers, determined by the derivative $d \mathrm{Fr} / d x$, should have a high value, which will avoid sections with an unstable flow structure. At the entrance and exit of the flume, the gradients of Froude numbers should be equal to zero $d \mathrm{Fr} / d x=0$, which will ensure a smooth entrance of the flow to the flume and its smooth exit into the downstream channel. In general, the measures make it possible to build the Venturi channel smoothly streamlined - "ideal".

\subsection{Longitudinal profiles of smoothly streamlined Venturi flumes}

The smoothly streamlined ("ideal") longitudinal profile of the water meter Venturi flume can be constructed by specifying a continuous monotonically increasing function $\operatorname{Fr}(x)$, which determines the nature of the change in Froude numbers along the flow direction. The function $\operatorname{Fr}(x)$ must correspond to the following boundary conditions: at the entrance to the Venturi flume at $x=0$, its value is determined by the equality (16) or the identical to it (19)

$$
\operatorname{Fr}_{u}=m \sqrt{2}=2 \sqrt{2 \frac{B}{b_{c}}} \sin ^{1.5}\left[\frac{1}{3} \arcsin \left(\frac{b_{c}}{B}\right)\right]
$$

and its derivative is equal to zero $(d \mathrm{Fr} / d x=0)$; at the end of the tray at $x=L$ (where $L$ is the length of the tray), the value of the function corresponds to formula (17)

$$
\mathrm{Fr}_{d}=2 \sqrt{2 \frac{B}{b_{c}}} \cos ^{1.5}\left[\frac{1}{3} \arccos \left(-\frac{b_{c}}{B}\right)\right]
$$

and its derivative is also zero $(d \mathrm{Fr} / d x=0)$; in the gorge cross-section $\mathrm{Fr}=1$ and $d \mathrm{Fr} / d x>0$.

An additional condition is a smooth streamlining the diffuser walls of the Venturi channel. According to the Russian State Standard MI 2406-97, the tangent of the angle of the expansion of the Venturi channel walls in the plan (cf. Fig. 2) be to no more than $1 / 6$. This sets the maximum value of the derivative $d b / d x$ not more than $1 / 3$, which is easily achieved by selecting the length of the Venturi flume L.

Based on the boundary conditions, the function $\operatorname{Fr}(x)$ can be specified by a polynomial or a trigonometric function.

Let us write down the polynomial of the third degree 


$$
\mathrm{Fr}=a_{0}+a_{1} \frac{x}{L}+a_{2}\left(\frac{x}{L}\right)^{2}+a_{3}\left(\frac{x}{L}\right)^{3}
$$

and its derivative

$$
\frac{d \mathrm{Fr}}{d x}=\frac{a_{1}}{L}+2 \frac{a_{2} x}{L^{2}}+3 \frac{a_{3} x^{2}}{L^{3}} .
$$

For $x=0$, according to the boundary conditions, we obtain

$$
\begin{aligned}
& \mathrm{Fr}=\mathrm{Fr}_{u}=a_{0}, \\
& \frac{d \mathrm{Fr}}{d x}=0=\frac{a_{1}}{L},
\end{aligned}
$$

therefore: $a_{0}=\mathrm{Fr}_{u}$ and $a_{1}=0$.

For $x=L$, we find

$$
\begin{gathered}
\mathrm{Fr}=\mathrm{Fr}_{d}=\mathrm{Fr}_{u}+a_{2}+a_{3}, \\
\frac{d \mathrm{Fr}}{d x}=0=2 \frac{a_{2}}{L}+3 \frac{a_{3}}{L},
\end{gathered}
$$

hence, according the second equality, we have $a_{3}=-2 a_{2} / 3$, and from the first equality we get $a_{2}=3\left(\mathrm{Fr}_{d}-\mathrm{Fr}_{u}\right)$ and $a_{3}=-2\left(\mathrm{Fr}_{d}-\mathrm{Fr}_{u}\right)$. Thus, as a result, the desired function takes the form

$$
\mathrm{Fr}=\mathrm{Fr}_{u}+\left(\mathrm{Fr}_{d}-\mathrm{Fr}_{u}\right)\left(3-2 \frac{x}{L}\right)\left(\frac{x}{L}\right)^{2}
$$

The function $\operatorname{Fr}(x)$ given by the polynomial (26) is shown in Fig. 5 on the left.

As the trigonometric function Fr (x), it is convenient to use the cosine function, which, with the arguments equal to 0 and $\pi$, has a derivative value equal to zero. It is easy to establish that the average line of this function must pass above the axis of abscissas at an altitude of $0.5\left(\mathrm{Fr}_{u}+\mathrm{Fr}_{d}\right)$, and the amplitude of the function should be equal to $0.5\left(\mathrm{Fr}_{u}-\right.$ $\mathrm{Fr}_{d}$ ). Following this logic, we will write down

$$
\mathrm{Fr}=0.5\left(\mathrm{Fr}_{u}+\mathrm{Fr}_{d}\right)+0.5\left(\mathrm{Fr}_{u}-\mathrm{Fr}_{d}\right) \cos \left(\pi \frac{x}{L}\right)
$$

and

$$
\frac{d \mathrm{Fr}}{d x}=\frac{\pi}{2 L}\left(\mathrm{Fr}_{d}-\mathrm{Fr}_{u}\right) \sin \left(\pi \frac{x}{L}\right)
$$

Indeed, for $x=0$ we have: $\mathrm{Fr}=\mathrm{Fr}_{u}$ and $d \mathrm{Fr} / d x=0$, and for $x=L: \operatorname{Fr}=\mathrm{Fr}_{d}$ and $d \mathrm{Fr} / d x=$ 0 . The function $\operatorname{Fr}(x)$ given by Eq. (27) is shown in Fig. 5 on the right. 

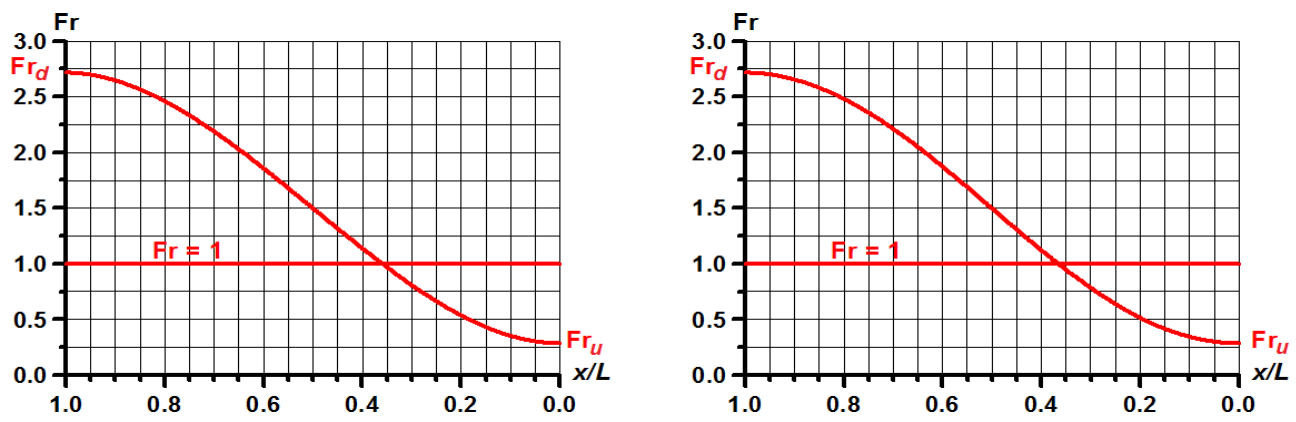

Fig. 5. Scheme for calculating the functions $\operatorname{Fr}(x)$ : on the left - the cubic polynomial, on the right the trigonometric function.

After calculating the functions $\operatorname{Fr}(x)$, then according to (11) the longitudinal profile of the Venturi channel is constructed (Fig. 6).

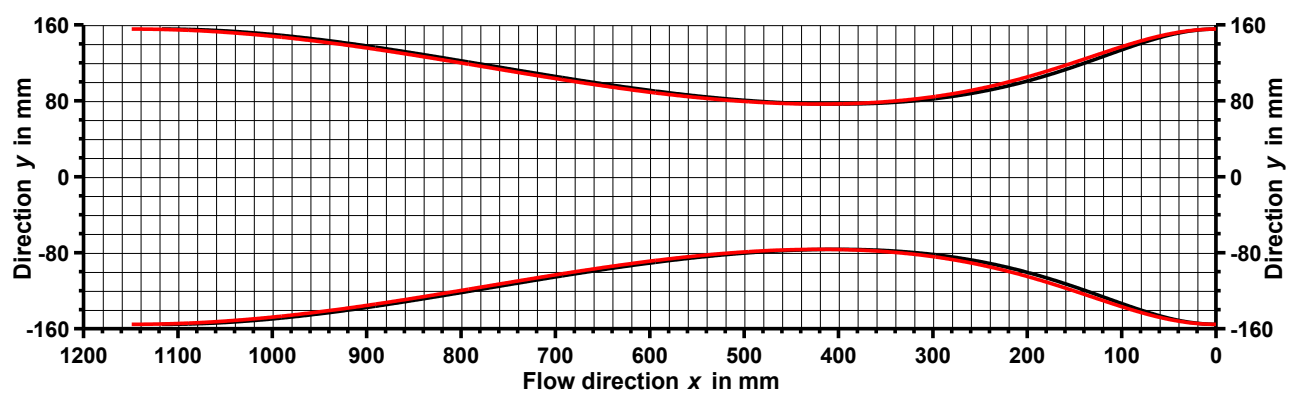

Fig.6. Longitudinal profiles of the Venturi channels: by the polynomial (26) - the black graph, by the function (27) - the red graph.

By the graphs Fig. 6, it is easy to make sure that both versions of the flume with dimensions $\mathrm{B}=311 \mathrm{~mm}$ and $b_{c}=153 \mathrm{~mm}$ practically equally ensure the smoothness of the Venturi channel profile in plan. However, the profile $b(x)$, given by the function $\operatorname{Fr}(x)$ through the cosine, is somewhat more extended along the longitudinal coordinate, that is, it is more smoothly varying. This reflects the above requirements. Thus, the profile given by the cosine function is adopted as optimal. In Fig. 7 shows the hydraulic characteristics of the flow in the Venturi channel of the optimal profile.

Comparison of the graphs in Fig. 7 and Fig. 3 allows us to conclude that in the Venturi channel of the calculating optimal longitudinal profile the flow characteristics along the flume change much more smoothly than in the investigated one. Properly speaking, this was our purpose, which ensures the creation of a flow with negligible hydraulic losses and with guaranteed, amenable to analytical calculation, hydraulic characteristics.

\subsection{Calculation example}

Let us dwell on the method of selection of the Venturi flume. Let it be given: the maximum flow rate $Q$ of clean water or wastewater, the width of upstream channel $B$ and the permissible water level in it $H$ (geometric head). In this case, by formulas (18) and (19), the flow coefficient $m$ of the required Venturi flume, the Froude number $\operatorname{Fr}_{u}$ in the upstream cannel can be determined

$$
\frac{Q}{B \sqrt{2 g} H^{1.5}}=m=\sqrt{0.5} \mathrm{Fr}_{u}
$$


and, according to (11), the ratio of the width of the critical cross-section $b_{c}$ to the width $B$

$$
\frac{b_{c}}{B}=\sqrt{\frac{27 \alpha_{0} \mathrm{Fr}_{u}^{2}}{\left(\alpha_{0} \mathrm{Fr}_{u}^{2}+2\right)^{3}}} .
$$

From the last relation the width of the critical cross-section $b_{c}$ is calculated.

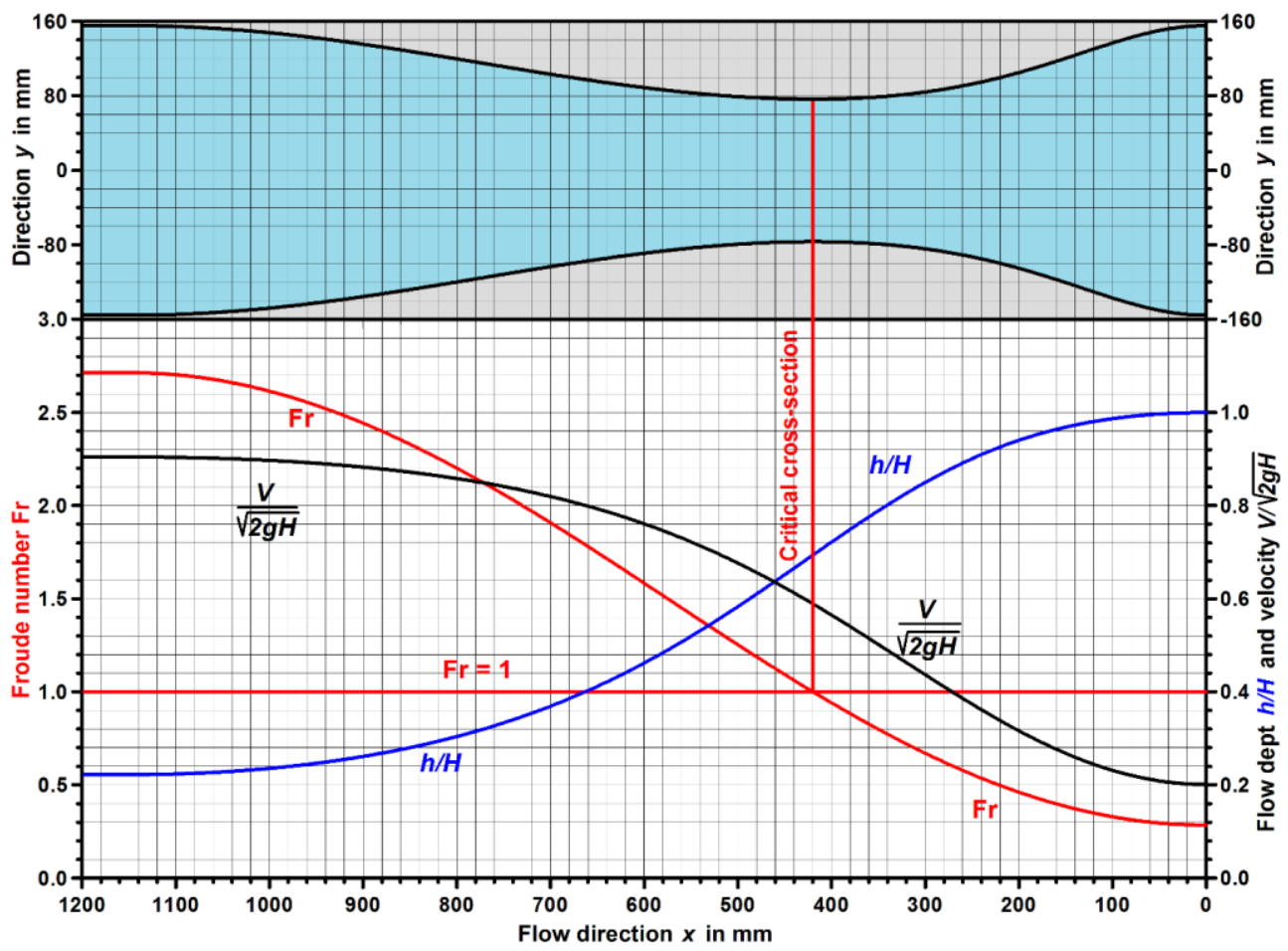

Fig. 7. Changing of depths, velocities and Froude numbers along the flow direction in the Venturi channel of the optimum profile.

For example, let the maximum flow rate of the Venturi flume be equal to $Q=300 \mathrm{~m}^{3} / \mathrm{h}$, the width of the upstream channel is $B=350 \mathrm{~mm}$, and the maximum level of its filling should be no higher than $H=400 \mathrm{~mm}$. We calculate

$$
m=\frac{Q}{B \sqrt{2 g} H^{1.5}}=\frac{300}{3600 \cdot 0.35 \cdot \sqrt{2 \cdot 9.81} \cdot 0.4^{1.5}}=0.2125
$$

and

$$
\mathrm{Fr}_{u}=m \sqrt{2}=0.2125 \sqrt{2}=0.3005
$$

Assuming the Boussinesq coefficient to be equal to $\alpha_{0}=1$ (see above), we find

$$
\frac{b_{c}}{B}=\sqrt{\frac{27 \alpha_{0} \mathrm{Fr}_{u}^{2}}{\left(\alpha_{0} \mathrm{Fr}_{u}^{2}+2\right)^{3}}}=\sqrt{\frac{27 \cdot 0.3005^{2}}{\left(0.3005^{2}+2\right)^{3}}}=0.5167
$$

and $b_{c}=0.5167 B=0.5167 \cdot 0.35=0.1808 \mathrm{~m}$.

The subsequent calculation, including the calculation of the longitudinal profile and all 
main hydraulic characteristics of the optimum Venturi flume, is carried out to the procedure described in the article.

\section{Conclusions}

1. It has been established that with the "dynamic" approach, based on the equation of momentum of forces, and with the classical "energetic" approach, based on the Bernoulli and Charnomsky equations, the differential equation of the smoothly changing fluid movement in the non-prismatic open channel remains the same.

2. A functional relationship is established between the Froude number for the flow in an arbitrary cross-section of the Venturi channel and its width, which is determined by the analytical formula (11).

3. It is established that within the rectilinear gorge of a classical not flooded Venturi channel with a horizontal bottom and vertical walls, the flow is unstable. This phenomenon is related to the proximity of the flow parameters to the critical ones with Froude numbers from $\mathrm{Fr}=0.85$ to $\mathrm{Fr}=1.15$. Thus, the presence of a long straight-line gorge distorts the hydraulics of the water meter channel.

4. By analogy with pressurized water-measuring nozzles of the Venturi, we find it expedient to perform flow meters Venturi for open channels with critical cross-section at a gorge as a dividing cut. In the gorge, when the flow passes through $\mathrm{Fr}=1$, the increase of Froude numbers, determined by the derivative $\mathrm{dFr} / \mathrm{dx}$, should have a high value, which will avoid sections with an unstable flow structure. At the entrance and exit of the flume, the gradients of Froude numbers should be equal to zero $\mathrm{dFr} / \mathrm{dx}=0$, which will ensure a smooth entrance of the flow to the flume and its smooth exit into the downstream channel. In general, the measures make it possible to build the Venturi channel extremely streamlined - "ideal".

5. A method has been developed for optimizing the longitudinal profile of vertical walls of the Venturi flow meter channel, which ensures the creation of a flow with negligible hydraulic losses and with guaranteed, amenable to analytical calculation, hydraulic characteristics. The method does not contain empirical coefficients and is based on computational and analytical studies with experimental verification.

6. The developed method allows to perform analytical calculations of all main hydraulic characteristics of the Venturi flume, such as the flow coefficient, the distributions of Froude numbers, of normalized depths and flow velocities along the length of the Venturi channel. The initial parameter for the calculations is the ratio of the width of the critical section $b_{c}$ in the gorge to the width of the upstream channel $B$. Thus, the ratio $b_{c} / B$ determines both the longitudinal profile of the Venturi tray and all its hydraulic characteristics.

7. When refining or revising the current State Standard of Russian Federation MI 2406-97, we find it expedient to include in its new edition the analytical method of hydraulic calculation of the Venturi channel of critical depth, discussed in the article, taking its relative error $\pm 1 \%$.

\section{References}

1. U.A. Vjazmin, V.N. Korneev, O.D. Loitsker, M.A. Mordjasov, V.I. Nikitin, M.N. Shafranovsky, Water Supply and Sanitary Technique 8, 76-78 (2013)

2. O.D. Loitsker, Water Supply and Sanitary Technique 3, 70-73 (2014)

3. N.L. Yegorov, O.D. Loitsker, M.N. Shafranovsky, Water Supply and Sanitary Technique 1, 66-71 (2016)

4. Z. Samani, H. Magallanez, J. Irrigation and Drainage Eng. 126(2), 127-129 (2000) 
5. O. Castro-Orgaz, J. Hyd. Res. 46, 691-698 (2008)

6. O. Castro-Orgaz, H. Chanson, Environmental Fluid Mech. 11, 499-516 (2011)

7. H. Chanson, Flow Measurement and Instrumentation 29, 65-66 (2013)

8. M. Dufresne, J. Vazquez, J. Hyd. Res. 51, 465-468 (2013)

9. E.D. Farsirotou, S.I. Kotsopoulos, Environmental Processes 2, 133-139 (2015)

10. D.J. Howes, C.M. Burt, B.F. Sanders, J. Irrigation and Drainage Eng. 136, 617-626 (2010)

11. S.W. Igo, K.N'wuitcha, K. Palm, L. Mihaescu, D.J. Bathiébo, Adv. in App. Sci. Res. 5(2), 359-367 (2014)

12. M.F. Maghrebi, J.E. Ball, J. Hyd. Eng. 132-10, 1044-1051 (2006)

13. V.B. Raskar, Int. J. Sci. Res. Tech. 3-2, 18-21 (2017)

14. N.L. Yegorov, O.D. Loitsker, Water Supply and Sanitary Technique 6, 70-74 (2012)

15. Y.T. Zerihun, Fluids 1(3), 21 (2016)

16. A.L. Zuikov, V.V. Bakunjaeva, Hydraulic Eng. 9, 47-56 (2017)

17. A.L. Zuikov, V.V. Bakunjaeva, Power Tech. Eng. 51-6, 621-627 (2018)

18. A.L. Zuikov, V.V. Bakunjaeva, T.V. Artemyeva, E.Yu. Zhazha, Magazine of Civil Engineering 2, 76-90 (2018)

19. A.D. Girgidov, Proc. B.E. Vedeneev All Russia Inst. Hyd. Eng. 248, 122-128 (2007)

20. A.D. Girgidov, Power Tech. Eng. 47-5, 344-347 (2014)

21. Yu.V. Bryanskaya, Power Tech. Eng. 44-2, 103-108 (2010)

22. N.K. Yerzhanova, Zh.A. Mussin, S.K. Dzholdasov, A. Altynbekova, Magazine of Civil Engineering 8, 106-114 (2017)

23. G.A. Korn, T.M. Korn, Mathematical Handbook for Scientists and Engineers (Dover Publications, 2000) 